\title{
6-minute walk test as a measure of disease progression and fatigability in a cohort of individuals with RYR1-related myopathies
}

Jessica W. Witherspoon ${ }^{1 *}$ (D, Ruhi P. Vasavada ${ }^{2}$, Melissa R. Waite ${ }^{2}$, Monique Shelton ${ }^{1}$, Irene C. Chrismer ${ }^{1}$, Paul G. Wakim ${ }^{4}$, Minal S. Jain², Carsten G. Bönnemann ${ }^{3}$ and Katherine G. Meilleur ${ }^{1}$

\begin{abstract}
Background: RYR1-related Myopathies (RYR1-RM) comprise a group of rare neuromuscular diseases (NMDs) occurring in approximately 1/90000 people in the US pediatric population. RYR1-RM result from pathogenic mutations in the ryanodine receptor isoform-1 (RYR1) gene where consequent RyR1 protein calcium dysregulation leads to impaired excitation-contraction coupling, oxidative and nitrosative stress, and mitochondrial depletion. These physiological deficits perpetuate RyR1 dysfunction causing further muscle injury, muscle weakness, and muscle fatigue. Muscle weakness and fatigue are two primary complaints in patients with RYR1-RM and are major symptoms that limit the ability of individuals to perform activities of daily living. The six-minute walk test (6MWT) is an endurance test with high reliability and validity used to measure walking capacity, disease progression, and more recently, fatigability in NMDs with limited results in RYR1-RM. Therefore, the purpose of our study is to objectively assess disease progression and fatigability in RYR1-RM affected individuals using the 6MWT. We hypothesized that 6MWT distance and fatigability would not change significantly between 0 and 6-month visits in RYR1-RM patients, given the clinically reported stable or slowly progressive nature of the disease. We also hypothesized participants would show fatigability during the 6MWT, given muscle weakness and fatigue are the two primary complaints of affected individuals.
\end{abstract}

Results: As expected, paired t-test analyses revealed no significant difference between total distance traveled $(p=.608)$ or percent change in speed $(p=.141)$ at 0 -months compared with the 6 -month visit. Fatigability was observed given the decline in speed between the first and last minute of the $6 \mathrm{MWT}$ at the 6 -month time point $(p \leq .0005$ ). Although this decline was not significant at baseline, a significant decline in speed from the 1st minute did occur at minutes 2,3 , and 4 during the baseline visit.

Conclusion: In this RYR1-RM cohort, the 6MWT showed disease stability over a 6-month period but revealed fatigability during the test. Given these results, the $6 \mathrm{MWT}$ may be a promising endpoint for evaluating fatigability and therapeutic efficacy in the 6-month treatment phase of our current n-acetylcysteine trial in this population. Improvement post intervention could be attributed to the intervention rather than variability in disease progression.

Trial Registration: Clinical Trials.gov, NCT02362425, Registered 13 February 2015-Prospectively registered.

Keywords: RyR1, 6-min walk test, Disease progression, Fatigue

\footnotetext{
* Correspondence: jessica.witherspoon@nih.gov

${ }^{1}$ National Institute of Nursing Research, NIH, Bethesda, MD, USA

Full list of author information is available at the end of the article
}

(c) The Author(s). 2018 Open Access This article is distributed under the terms of the Creative Commons Attribution 4.0 International License (http://creativecommons.org/licenses/by/4.0/), which permits unrestricted use, distribution, and reproduction in any medium, provided you give appropriate credit to the original author(s) and the source, provide a link to the Creative Commons license, and indicate if changes were made. The Creative Commons Public Domain Dedication waiver (http://creativecommons.org/publicdomain/zero/1.0/) applies to the data made available in this article, unless otherwise stated. 


\section{Background}

Affecting 1/90,000 children in the United States (US) [1], RYR1-related myopathies (RYR1-RM), though rare, comprise the most common congenital myopathies [2] in the US. RYR1 is one of the largest genes in the human genome and encodes the major calcium channel in skeletal muscle, ryanodine receptor isoform-1 (RyR1). Numerous variants in RYR1 have been identified as the cause of RYR1-RM subtypes, including central core disease $(\mathrm{CCD})$, multi-mini core disease $(\mathrm{MmD})$, centronuclear myopathy (CNM), core-rod myopathy, and congenital fiber type disproportion (CFTD).

RYR1 mutations yield RyR1 protein dysfunction and thus calcium dysregulation, resulting in impaired excitation-contraction coupling and excessive mitochondrial oxidative stress [3, 4]. Affected individuals present with combinations of delayed motor milestones, hypotonia, fatigue, extremity muscle weakness, joint contractures, progressive scoliosis, susceptibility to malignant hyperthermia $(\mathrm{MH})$, and, in more severe cases, ophthalmoplegia and/or respiratory failure $[3,5,6]$. RYR1-R$\mathrm{M}$-associated symptoms may impair quality of life, especially in severely affected individuals, who are at risk for early mortality. Muscle weakness and fatigue are the two primary complaints in patients with RYR1-RM and are major symptoms limiting the ability of individuals to perform instrumental activities of daily living [7-9]. De Vries et al. noted [8], "fatigue accounts for an important part of the burden experienced by patients with neuromuscular disorders [8]."

The 6-min walk test (6MWT) is an endurance test with high reliability and validity [10] that allows for the assessment of walking capacity, disease progression, and treatment efficacy [11]. Recently [10, 11], the 6MWT was shown to serve as a measure of fatigability in neuromuscular diseases (NMDs) as determined by a decline in speed $[10,11]$. Andersen et al. observed a $4.6 \%$ decline in speed (meters/second) between first minute and last minute (minute 6) of the 6MWT in patients with various NMDs [11]. Currently, the 6MWT is not only the preferred measure of walking capability and endurance, but also of fatigability in adult and pediatric neurological and neuromuscular disorders [12]. It has been used as the primary outcome measure in clinical trials, which led to its approval as one of the preferred walking test measurements $[13,14]$. However, the 6MWT has not yet been objectively studied as an outcome measure in RYR1-RM affected individuals.

The clinical impression of RYR1-RM suggests a non-progressive (static) $[15,16]$ or slowly progressive [16] group of diseases. However, disease progression has likewise not been formally studied. It remains undetermined whether RYR1-RM would remain stable or progress over the time frame of a typical clinical trial. The purpose of our study was to objectively assess disease progression and fatigability in individuals with RYR1-RM using the $6 \mathrm{MWT}$ over 6 months, in preparation for future clinical trials.

\section{Results \\ 6MWT \\ Disease progression}

RYR1-RM participants, on average, performed $~ 79 \%$ (range 32.2-119\%) of the predicted norm for their age, sex, and height on the 6MWT [17-19]. This average did not change between the baseline $(79.1 \% \pm 3.55)$ and 6 -month $(79.9 \% \pm 3.72)$ visits. The mean distances walked at baseline $(477 \mathrm{~m} \pm 22.8)$ and 6 months $(481 \mathrm{~m}$ $\pm 23.2)$ were also compared. Paired t-test analysis showed there was no evidence of change in percent of the predicted norm $(p=.612)$ or distance walked between visits $(p=.608)$. Similarly, the percent change in walking speed between the first and 6th minute did not change between visits; $p=.141$. Figure 1a compares total distance walked at baseline and 6-month visits per individual. For each visit, the mean walking speed and the mean percent change in walking speed between minutes 1 and 6 are shown in Fig. 1b.

\section{Fatigability}

Paired t-test analyses revealed no significant difference between percent change in speed between the 0-month and 6-month visits. However, a decline in speed between the first and last minute of the 6MWT was observed. As depicted in Fig. 2a, mean walking speeds during the $6 \mathrm{MWT}$ were variable at the baseline visit: the average speed started to plateau after the 2-min interval, increased at the 5-min interval, and then returned to the 2-min interval speed. In contrast, mean walking speeds declined and gradually plateaued throughout the test when performed at 6 months.

At the baseline visit, the fastest walking speed was in the first minute. Minutes 2-6 showed variations in speed, with the slowest minute being minute 4. Although walking speed was variable during the baseline visit, the speeds at minutes $2(p=.003), 3(p=.006)$, and $4(p=.0001)$ were each significantly slower than the speed walked in the first minute. These $p$-values are unadjusted for multiplicity.

Similar to the baseline visit, the 1st minute of the 6-month visit $6 \mathrm{MWT}$ was the fastest. After the first minute a plateau in speed was observed (Fig. 2b). There was a significant $(p \leq .0005)$ decrease in walking speed between the first minute and each minute interval thereafter (mins 2-6). As expected, the observed change (decrease) in distance traveled between the first and last minute of the 6MWT was reflected in the change 

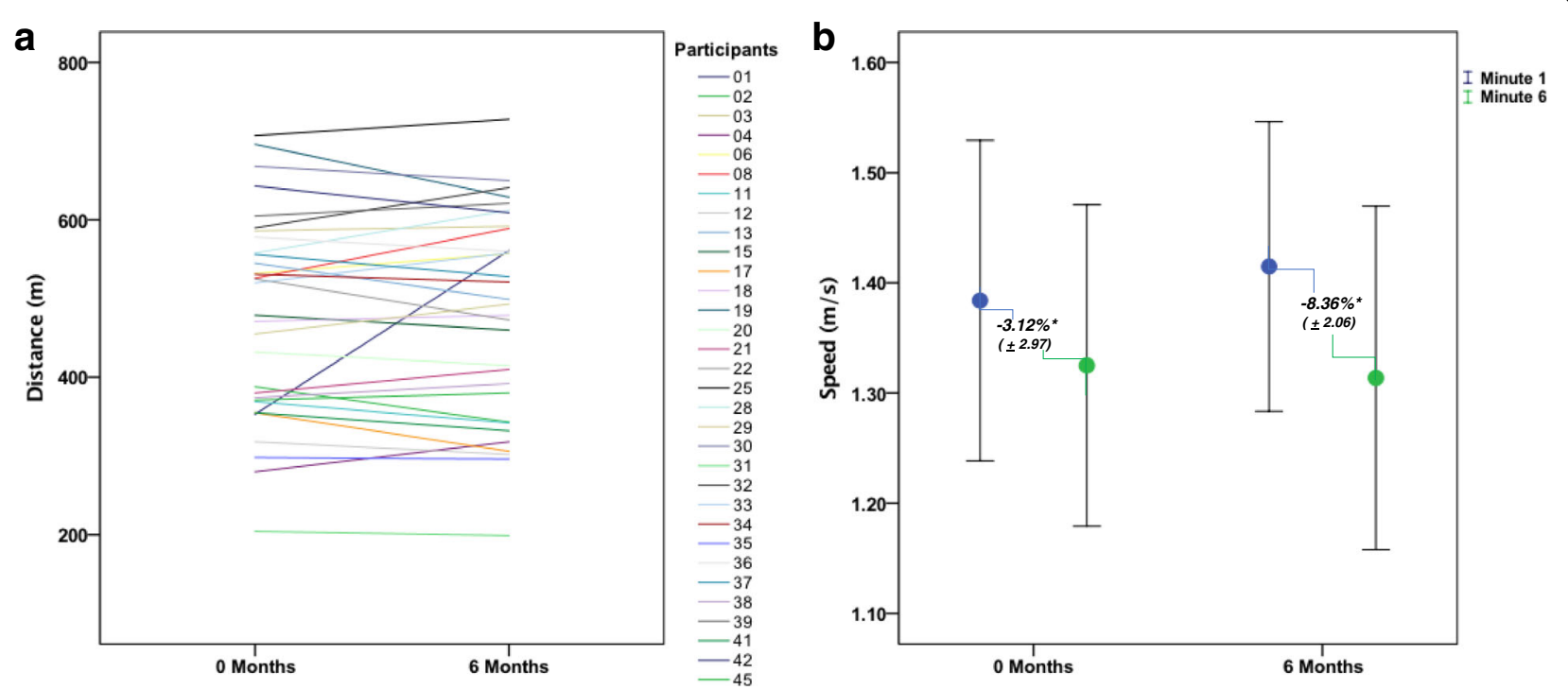

Fig. 1 Disease Progression. a Total 6-min walk distance at 0 and 6-month visits by participant. This figure includes only the 32 participants with both 0 - and 6-month data. b Mean walk speed $(95 \% \mathrm{Cl})$ at minutes 1 and 6 at 0 and 6 month visits. Percentage values represent the \% change between minute 1 and minute 6 for each visit. *significance

(decline) in speed between the first and last minute of the $6 \mathrm{MWT}$.

The speeds of adults and children were also compared. At both month 0 and month 6 , the average speeds across the $6 \mathrm{~min}$ were not statistically different between the two age groups (month $0, p=.782$; month $6, p=.389$ ). Only at minute 5 of month 0 , did the average speed of children exceed that of adults, hence the interaction between minute intervals and age group (Fig. 2c). In all other minutes and in both month 0 and 6 , the speed for adults was slightly higher than that of children, but again the difference was not statistically significant (Fig. 2c and d).

Interestingly, the speed at the 2-min interval was comparable to the speed at the 6-min interval at both study visits. The initial decline in speed from the first to the second minute was nearly comparable to the decline in speed from the 1st-minute interval to the 6th-minute interval. In contrast, no significant difference was observed at either visit between the speed at $2 \mathrm{~min}$ and at 6 min (baseline visit: $p=.875,6$-month: $p=.161$ ). Given that researchers are currently assessing the possibility of implementing a 2-min walk test (2MWT) instead of the $6 \mathrm{MWT}$, fatigability in addition to endurance may be another important variable to assess during a $2 \mathrm{MWT}$ in the NMD population.

\section{Discussion}

The 6MWT originated as a way to measure functional capacity and endurance in people with cardiorespiratory disease, for which normative values have been established [20]. Now, the $6 \mathrm{MWT}$ is widely used across diseases, including
NMDs [11, 20-22]. Andersen et al. reported using 6MWD in NMDs, including myotonic dystrophy type 1 , facioscapulohumeral dystrophy, sporadic inclusion body myositis, Kennedy disease, Limb-girdle muscular dystrophy, Charcot Marie-Tooth, mitochondrial myopathy, and mixed myopathies. The average distance ambulated across all of the above-mentioned diseases was $405 \mathrm{~m}$ (range 65-750 m).

More recently, the $6 \mathrm{MWT}$ has also been employed as a measure of fatigability in multiple sclerosis (deceleration index depending on disease severity) [23], myasthenia gravis (degree of deviation from stable performance using linear trends) [24], and the aforementioned NMDs (percent decrease between 1st and 6th minute) [11]. Fatigability is defined as an objective measurement of decline in performance [24].

This natural history study assessed disease progression and fatigability in an RYR1-RM cohort over a 6-month time course in preparation for future clinical trials. Our study showed there was no significant change in the mean distance walked or mean walking speed during the 6MWT over a 6-month time frame, confirming the stable or slowly progressive nature of RYR1-RM, despite having a phenotypically heterogeneous cohort. Although this study was of shorter duration than most natural history studies, it provides a glimpse into the progression of RYR1-RM during a 6-month time frame. If an intervention were to show improvement in RYR1-RM within this time frame, this change could be attributed to the intervention rather than disease progression. A clinically meaningful difference of $28.5 \mathrm{~m}$ has been established in boys with Duchenne muscular dystrophy older than 5 years of age [25], but no such difference has been established in individuals affected with RYR1-RM. 

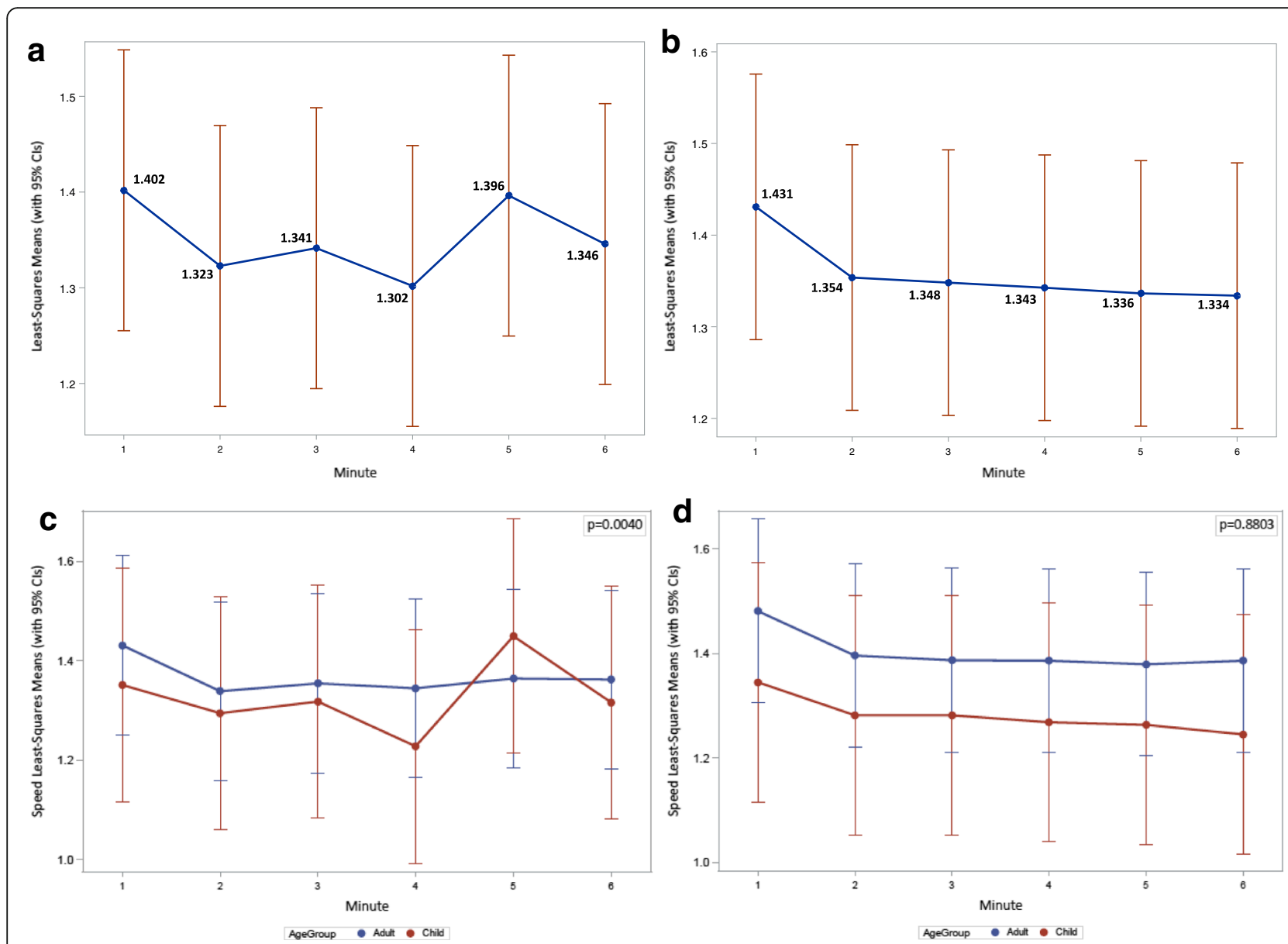

Fig. $\mathbf{2}$ Least square means for walking speed at each minute during 6MWT for baseline (a) and 6 month (b) visits. Comparison of least square means for walking speed at each minute during $6 \mathrm{MWT}$ between adults and children at baseline (c) and 6-month (d) visits

Given that our results demonstrated RYR1-RM disease stability using the 6MWT over a course of 6 months and this test is accepted as a clinically meaningful outcome measure in various NMDs [21], the 6MWT may serve as a promising endpoint for evaluating therapeutic efficacy in the treatment phase of current and future trials. Our results also indicate that walking speed may potentially be a clinically meaningful outcome in RYR1-RM. The decrease in walking speed observed in these RYR1-affected individuals between minutes 1 and 6 could be impacted by a treatment. In other diseases, an improvement of walking speed of $0.1-0.2 \mathrm{~m} / \mathrm{s}$ has been reported as the minimal clinically important difference (MCID) [26]. Using this information and our 6MWT data in RYR1-RM affected individuals, only 10 people would be needed to power a trial at $90 \%$ with an alpha of .05 if change in walking speed were the primary outcome based on an MCID of $0.2 \mathrm{~m} / \mathrm{s}$.

Compared with the general NMD population [11], participants with RYR1-RM had similar walking speeds to individuals with Becker Muscular Dystrophy (1st min:
$1.43 \mathrm{~m} / \mathrm{s}$, 6th $\min : 1.36 \mathrm{~m} / \mathrm{s})$. RYR1-RM affected individuals began the test with the fastest 6-min walk speed $(1.43 \mathrm{~m} / \mathrm{s})$, but also exhibited the greatest decline in speed $(1.33 \mathrm{~m} / \mathrm{s})$ at the end of the test, which was observable starting at the 2-min interval. In our cohort, individuals with RYR1-RM demonstrated variable speeds at minute intervals at the baseline visit. In contrast, at the 6-month visit, there was an initial decline in speed that gradually plateaued after the 2nd minute. The fastest speed was achieved in the first minute at both baseline and 6-month visits. Because the variability in speed was observed at baseline but not at the 6-month visit, this variability could be attributed to a learning effect, which is common in other NMDs $[27,28]$. Utilizing standardized 6-min walk testing methods, our findings demonstrated a decline in performance at both time points, which suggests that individuals with RYR1-RM exhibit fatigue during the 6MWT. A decline in speed between the first and 6th minute was also accompanied, as would be expected, by a decrease in distance traveled, reflecting a decline in performance by the end of the test. 
To the best of our knowledge, a single study has assessed the percent decline in NMDs compared with the healthy population to date [11]. However, standard error values were not provided, and thus we were unable to directly compare the average percent decline between their study and ours. The reported percent decline in walking speed for the group of NMDs (myotonic dystrophy, limb girdle muscular dystrophy, fascioscapulohumeral dystrophy, Kennedy disease, Charcot Marie-Tooth neuropathy, mitochondrial myopathy, sporadic inclusion body myositis, Becker muscular dystrophy, and a mixed group of myopathies including congenital myopathy) was $4.6 \%$ between the first and last minute [11]; individuals in our RYR1-RM cohort presented with a mean percent decline in speed of $3.12 \%$ $( \pm 2.97)$ at baseline and $8.36 \%( \pm 2.06)$ at the 6 -month visit. The previously reported healthy population demonstrated a $1.4 \%$ decline in walking speed over the 6 min [11]. Percent decrease in speed may be clinically meaningful in identifying fatigue-related functional capacity in RYR1-RM, but this would require a larger 6MWT study including both RYR1-RM and healthy participants.

There has been a growing interest in comparing the two-minute walk test with the 6MWT [29]. Interestingly, a decline in speed in our cohort initially occurred at the 2-min interval of the 6MWT for the baseline and 6 -month visits. This decline in speed from the first minute interval to the 2nd minute interval was not significantly different from the decline in speed between the first and 6th minute intervals. Given our study shows that fatigability can be captured within the first 2 min of the 6MWT, this may further support the use of a 2MWT but needs to be explored further.

Since the 6MWT detects a decline in walking speed in RYR1-RM, prospectively studying cardiorespiratory measures of fatigue during the 6MWT could provide insight into the cardiorespiratory response of individuals with RYR1-RM. In people with cystic fibrosis and chronic obstructive pulmonary disease, a decline in speed during the 6MWT with an increase in HR, oxygen saturation, and dyspnea has been observed [30]. However, cardiorespiratory fatigue response may not be achieved in people with RYR1-RM given that they do not have primary lung disease and local muscle fatigue may occur first in myopathy patients.

\section{Study limitations}

Given the rare nature of this disease, a few limitations to our study include the small sample size and varied disease expression among participants, which limits our ability to categorize by severity. Thus, we were unable to observe whether performances varied by disease severity or by the presence of neuromuscular issues that limited walking in some cases. We are currently exploring criteria for categorizing disease severity. Also, the differences in intermittent vs gradual decline in speed at the baseline versus 6-month visits could be due to a learning effect or to changes in individuals' efforts. This may be addressed in the future by having a practice 6MWT with a period of rest.

\section{Conclusion}

The 6-min walk test has been used to assess endurance, ambulatory capacity, and cardiopulmonary function for a range of diseases for many years. More recently, it has been used to assess fatigability in neuromuscular disorders. This study was the first to evaluate 6-min walk distances and speeds in individuals with RYR1-RM in order to assess disease progression and fatigability over a course of 6 months. Our findings confirm clinical reports of RYR1-RM as being a group of stable or slowly progressive diseases. The significant decrease in walking speed between interval minutes 1 and 6 observed at both baseline and 6-month visits indicates the 6MWT is an appropriate measure of fatigability in individuals with RYR1-RM. Based on our results and the ability to now properly power future studies using these data, assessing changes in speed during 6MWT intervals may serve as a valid outcome measure for use in future natural history studies and/or clinical trials, especially those addressing fatigability in this population.

\section{Methods}

\section{Participants}

Participants were enrolled in our RYR1-RM double-blind, placebo-controlled N-acetylcysteine (NAC) Trial (NCT02362425) at the National Institutes of Health (NIH). This clinical trial, consisting of both a natural history and a treatment phase, was approved by the NIH Combined Neurosciences Institutional Review Board. Participants (and/or caregivers) who agreed to participate signed informed consent forms (and assent if a minor under the age of 18 years). Recruitment for this trial began in January 2015 . The natural history phase (0-6 months) of the clinical trial, reported here, was completed by 32 participants (13 males, 19 females) between March 2015 and November 2016 at the NIH Clinical Center in Bethesda, MD. Figure 3 shows the Strengthening the Reporting of Observational Studies in Epidemiology (STROBE) flow diagram for participants included in this paper. Of these participants, 12 were children with a mean age of $10.1( \pm 2.70)$, and 20 were adults with a mean age of $39.4( \pm 12.2)$. The cohort was comprised of $\mathrm{CCD}, \mathrm{MmD}$, and $\mathrm{CNM}$, many with malignant hyperthermia susceptibility. Inclusion criteria for this study included $\geq 7$ years of age, ambulatory without assistive devices or orthotics, and confirmed genetic diagnosis of RYR1-RM. Muscle biopsy was required initially, but, after 


\section{STROBE flow diagram for NCT02362425 "Antioxidant Therapy in RYR1-Related Congenital Myopathy: Natural History Phase"}

Enrollment

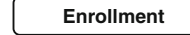

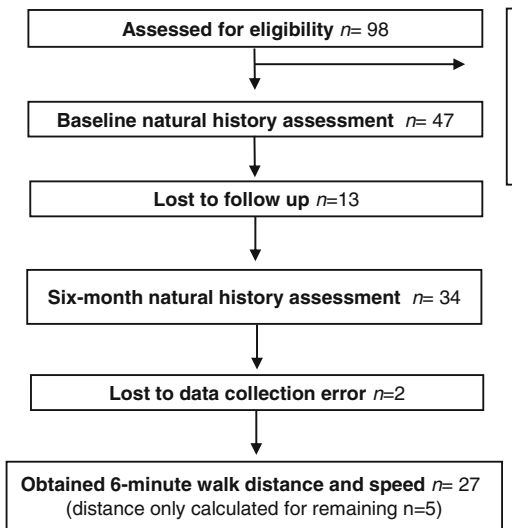

Excluded $n=57$

Genetic testing negative for RYR1-RM $n=7$

Unclear results from genetic testing $n=3$

No genetic testing on record $n=11$

Medical or medicinal contraindication $n=11$

Non-ambulatory $n=14$

Declined to participate $n=11$

(distance only calculated for remaining $n=5$ )

Fig. 3 STROBE Flow diagram for participants included in the natural history phase of the RYR1 NAC trial

six months, this criterion was changed to "preferred" to facilitate recruitment. Participants were excluded if they presented with a history of liver or lung disease, ulcers, dysphagia, were pregnant or breastfeeding, planned to become pregnant, or were consuming medicine that interacts with NAC or other antioxidants.

\section{MWT}

The $6 \mathrm{MWT}$ was administered in a single day at the NIH Rehabilitation Medicine Department using the American Thoracic Society (ATS) guidelines [14]. It was conducted at baseline in the early afternoon on the second day of the visit and was repeated at 6 months on the second day of the visit at the same time as baseline. After arrival at the testing site, participants rested for five minutes. Blood pressure and resting heart rate were measured before exercise, immediately post-exercise, and after a 5-min recovery. All participants were instructed to walk back and forth along a $50 \mathrm{~m}$ hallway for $6 \mathrm{~min}$, "as quickly and safely as possible." During the test, to ensure safety of the participant, a clinician trailed slightly behind the subject to avoid introducing a pacing bias. The participants were given a series of small objects (e.g., beanbag toys) to be used as markers. Participants were asked to drop the marker at every minute interval to record his/her location at the end of each minute. If dropping the markers appeared to be distracting to a participant, an individual who served as a chaser dropped the markers instead. Participants were not permitted to sit or lean against the wall or a chair if they required a rest period during the test. If rest was required, the clinician continued to keep time. Verbal encouragement was given in accordance with ATS guidelines [14]. In addition, children who appeared to be distracted or slowing were given more frequent verbal encouragement. The distance walked at each minute interval was documented. The setting, procedure, and clinician remained consistent when possible at the follow-up visit (6-months) to decrease potential sources of bias. In three cases, an alternate clinician administered the 6MWT.

\section{Statistics}

Data for the 6MWT were collected and stored in the NIH Clinical Trials Database, CTDB. Statistical analyses were performed using SAS v9.4 software. Descriptive analysis was used for sample demographics. Results are expressed as the mean \pm SEM (Standard Error of the Mean). Differences in $6 \mathrm{MWT}$ distance performed at 0 months (baseline) and 6 months as well as the percent change in walking speed at baseline and 6 months were determined using paired t-tests to assess disease progression. A repeated-measures mixed model was fit to the data to assess fatigability based on changes in walking speed per minute during the 6MWT. An age effect was also introduced into the mixed model to determine if there was a difference in performance between adults and children.

A total of 32 subjects completed the $6 \mathrm{MWT}$ at 0 and 6-month visits. Of the 32 participants, 3 did not have a distance recorded for the first minute during their baseline visit, so changes in walk speed and distance could not be determined. One participant did not have properly recorded four and five-minute interval distances during their second visit. However, the total 6-min walk distance (6MWD) was recorded. For this reason, all 32 participants data were included in statistics for disease progression as determined by total walked distance during 6MWT, but data from only 27 participants were used for walking speed and fatigability-related statistics.

\section{Abbreviations}

6MWD: 6-min walk distance; 6MWT: 6-min walk test; ATS: American Thoracic Society; CCD: Central core disease; CFTD: Congenital fiber type disproportion; CNM: Centronuclear myopathy; MH: malignant hyperthermia; MmD: Multi- 
mini core disease; NAC: N-acetylcysteine; NMDs: Neuromuscular diseases; RyR1: Ryanodine receptor isoform-1; RYR1-RM: RYR1-Related Myopathies

\section{Acknowledgements}

We thank Carmel Nichols and Janvi Raichura for assistance with data collection and storage. Fatou Tounkara for assisting with participant enrollment and Joshua Todd for creating the study flow diagram from which the STROBE diagram was created.

\section{Funding}

This work was supported by the National Institutes of Health (Bench-toBedside Award), National Institute of Nursing Research, Division of Intramural Research, and Office of Rare Disease. Funding was used for personnel, patient travel, lodging, meals, and statistical analyses. We thank the patients and families for their involvement in this study.

\section{Availability of data and materials}

Data for this study are stored in a secure online database, Clinical Trials DataBase (CTDB). May be made available with permission.

\section{Authors' contributions}

JW performed data management, data quality control, primary writer of manuscript, and reviewed/edited manuscript. RV assisted with 6MWT, performed data entry, assisted with writing manuscript, and reviewed/edited manuscript. MW administered 6-min walk test assessments, assisted with data quality control, and reviewed/edited manuscript. MS assisted with 6min walk test data management and storage. IC performed patient recruitment and enrollment for the study. PW performed statistics for the manuscript and reviewed/edited manuscript. MJ supervised research in the Rehabilitation Medicine Department at $\mathrm{NIH}$, administered 6-min walk test assessment when Melissa was unavailable, and reviewed/edited manuscript. CB performed final medical judgment for patient enrollment and reviewed/edited manuscript. KM is the PI of the study and reviewed/edited manuscript. All authors read and approved the final manuscript.

\section{Ethics approval and consent to participate}

This study (NCT02362425) and consent/assent forms were approved by the Institutional Review Board of the National Institutes of Health.

\section{Consent for publication}

Not applicable.

\section{Competing interests}

There are no conflicts of interest.

\section{Publisher's Note}

Springer Nature remains neutral with regard to jurisdictional claims in published maps and institutional affiliations.

\section{Author details}

${ }^{1}$ National Institute of Nursing Research, NIH, Bethesda, MD, USA. ${ }^{2}$ Rehabilitation Medicine, NIH, Bethesda, MD, USA. ${ }^{3}$ National Institute of Neurological Disorders and Stroke, NIH, Bethesda, MD, USA. ${ }^{4}$ Biostatistics and Clinical Epidemiology, NIH, Bethesda, MD, USA.

Received: 21 March 2018 Accepted: 20 June 2018

\section{Published online: 03 July 2018}

\section{References}

1. Amburgey K, McNamara N, Bennett LR, McCormick ME, Acsadi G, Dowling $J$ J. Prevalence of congenital myopathies in a representative pediatric United States population. Ann Neurol. 2011;70:662-5.

2. Illingworth MA, Main M, Pitt M, et al. RYR1-related congenital myopathy with fatigable weakness, responding to pyridostigimine. Neuromuscul Disord. 2014;24:707-12.

3. Jungbluth H, Sewry CA, Muntoni F. Core myopathies. Semin Pediatr Neurol. 2011;18:239-49.

4. Dowling JJ, Arbogast S, Hur J, et al. Oxidative stress and successful antioxidant treatment in models of RYR1-related myopathy. Brain. 2012;135:1115-27.

5. Bevilacqua JA, Monnier N, Bitoun M, et al. Recessive RYR1 mutations cause unusual congenital myopathy with prominent nuclear internalization and large areas of myofibrillar disorganization. Neuropathol Appl Neurobiol. 2011;37:271-84.

6. Zhou H, Jungbluth $\mathrm{H}$, Sewry CA, et al. Molecular mechanisms and phenotypic variation in RYR1-related congenital myopathies. Brain. 2007;130:2024-36.

7. Kluger BM, Krupp LB, Enoka RM. Fatigue and fatigability in neurologic illnesses: proposal for a unified taxonomy. Neurology. 2013;80:409-16.

8. de Vries JM, Hagemans ML, Bussmann JB, van der Ploeg AT, van Doorn PA. Fatigue in neuromuscular disorders: focus on Guillain-Barre syndrome and Pompe disease. Cell Mol Life Sci. 2010;67:701-13.

9. Kumor K, Pierzchala K. The problem of fatigue in neurological disorders. Wiad Lek. 2006;59:685-91.

10. Arafah A, Kuspinar A, Mayo N. Untangling perception of fatigue and fatigability: first steps. Austin J Multiple Sclerosis Neuroimmunology. 2015;2:1018-25.

11. Andersen LK, Knak KL, Witting N, Vissing J. Two- and 6-minute walk tests assess walking capability equally in neuromuscular diseases. Neurology. 2016;86:442-5.

12. Strauss N, Montes J, Russman B, et al. Rehabilitation of the child with a neuromuscular disorder. In: Darras B, editor. Neuromuscular disorders of infancy, childhood, and adolescence. 2nd ed. Oxford: Elsevier; 2015. p. 1070-89. eds.

13. Holland AE, Spruit MA, Troosters T, et al. An official European Respiratory Society/American Thoracic Society technical standard: field walking tests in chronic respiratory disease. Eur Respir J. 2014:44:1428-46.

14. Laboratories ATSCPSCPF. ATS statement: guidelines for the six-minute walk test. Am J Respir Crit Care Med. 2002;166:111-7.

15. Monnier N, Romero NB, Lerale J, et al. An autosomal dominant congenital myopathy with cores and rods is associated with a neomutation in the RYR1 gene encoding the skeletal muscle ryanodine receptor. Hum Mol Genet. 2000;9:2599-608.

16. Jungbluth H. Multi-minicore Disease. Orphanet J Rare Dis. 2007;2:31.

17. Chetta A, Zanini A, Pisi G, et al. Reference values for the 6-min walk test in healthy subjects 20-50 years old. Respir Med. 2006;100:1573-8.

18. Enright $\mathrm{PL}$, Sherrill DL. Reference equations for the six-minute walk in healthy adults. Am J Respir Crit Care Med. 1998;158:1384-7.

19. Geiger R, Strasak A, Treml B, et al. Six-minute walk test in children and adolescents. J Pediatr. 2007:150:395-9. 399 e1-2

20. Goemans N, Klingels K, van den Hauwe M, et al. Six-minute walk test: reference values and prediction equation in healthy boys aged 5 to 12 years. PLoS One. 2013;8:e84120.

21. Goemans N, van den Hauwe M, Wilson R, van Impe A, Klingels K, Buyse G Ambulatory capacity and disease progression as measured by the 6-minutewalk-distance in Duchenne muscular dystrophy subjects on daily corticosteroids. Neuromuscul Disord. 2013;23:618-23.

22. Lachmann $R$, Schoser $B$. The clinical relevance of outcomes used in lateonset Pompe disease: can we do better? Orphanet J Rare Dis. 2013;8:160.

23. Kopciuch F. Walking fatigability in multiple sclerosis: a comparison between the 2- and the 6-minute walk test and an evaluation of associated factors. Ann Phys Rehabil Med. 2016;59S:e43.

24. Jordan B, Mehl T, Schweden TL, Menge U, Zierz S. Assessment of physical fatigability and fatigue perception in myasthenia gravis. Muscle Nerve. 2017; 55:657-663.

25. McDonald CM, Henricson EK, Abresch RT, et al. The 6-minute walk test and other clinical endpoints in duchenne muscular dystrophy: reliability, concurrent validity, and minimal clinically important differences from a multicenter study. Muscle Nerve. 2013:48:357-68.

26. Bohannon RW, Glenney SS. Minimal clinically important difference for change in comfortable gait speed of adults with pathology: a systematic review. J Eval Clin Pract. 2014:20:295-300.

27. Knak KL, Andersen L, Witting N, Vissing J. Test-retest reliability of the 2- and 6-minute walk tests in patients with neuromuscular diseases. Neuromuscul Disord. 2015;25:s273

28. Prahm KP, Witting N, Vissing J. Decreased variability of the 6-minute walk test by heart rate correction in patients with neuromuscular disease. PLoS One. 2014;9:e114273.

29. Bohannon RW, Bubela D, Magasi S, et al. Comparison of walking performance over the first 2 minutes and the full 6 minutes of the sixminute walk test. BMC Res Notes. 2014;7:269.

30. Reychler G, Debatisse M, Lebecque P, Pieters T, Liistro G, Gohy S. Variability of gait speed during six minutes walking test in COPD and cystic fibrosis patients. Gait Posture. 2016;49:36-40. 